\title{
The importance of a multidisciplinary approach to hepatocellular carcinoma
}

\author{
Osama Siddique' \\ Eric R Yoo ${ }^{2}$ \\ Ryan B Perumpail ${ }^{3}$ \\ Brandon J Perumpail ${ }^{4}$ \\ Andy Liu ${ }^{5}$ \\ George Cholankeril ${ }^{6}$ \\ Aijaz Ahmed ${ }^{3}$ \\ 'Department of Medicine, \\ Brown University, Providence, \\ RI, ${ }^{2}$ Department of Medicine, \\ University of Illinois College of \\ Medicine, Chicago, IL, ${ }^{3}$ Division of \\ Gastroenterology and Hepatology, \\ Stanford University School of \\ Medicine, Stanford, CA, ${ }^{4}$ Department \\ of Medicine, Drexel University \\ College of Medicine, Philadelphia, PA, \\ ${ }^{5}$ Department of Medicine, California \\ Pacific Medical Center, San Francisco, \\ CA, ${ }^{6}$ Division of Gastroenterology \\ and Hepatology, University of \\ Tennessee Health Sciences Center, \\ Memphis, TN, USA
}

This article was published in the following Dove Press journal: Journal of Multidisciplinary Healthcare 20 March 2017

Number of times this article has been viewed

\begin{abstract}
Hepatocellular carcinoma (HCC) is the third leading cause of cancer-related deaths worldwide. The rising incidence, genetic heterogeneity, multiple etiologies, and concurrent chronic liver diseases make diagnosis, staging, and selection of treatment options challenging in patients with HCC. The best approach to optimize the management of HCC is one that utilizes a core multidisciplinary liver tumor board, consisting of hepatologists, pathologists, interventional radiologists, oncologists, hepatobiliary and transplant surgeons, nurses, and general practitioners. In most cases, HCC is diagnosed by abdominal imaging studies, preferably with a triphasic computed tomography scan of the abdomen or magnetic resonance imaging of the abdomen. Histopathological diagnosis using a guided liver biopsy may be needed in noncirrhotic patients or when radiological diagnostic criteria are not fulfilled in the setting of cirrhosis. The Barcelona Clinic Liver Cancer staging system facilitates a standardized therapeutic strategy based on the tumor burden, extent of metastasis, severity of hepatic decompensation, comorbid medical illnesses, functional status of patient, HCC-related symptoms, and preference of the patient. Treatment options include curative surgery (hepatic resection and liver transplantation) and palliative measures (radiofrequency ablation, transarterial chemoembolization, and chemotherapy with sorafenib). The role of the multidisciplinary team is crucial in promptly reconfirming the diagnosis, staging the HCC, and formulating an individualized treatment plan. In potential liver transplant candidates, timely liver transplant evaluation and coordinating bridging/downsizing treatment modalities, such as radiofrequency ablation and transarterial chemoembolization, can be time-consuming. In summary, a multidisciplinary team approach provides a timely, individualized treatment plan, which can vary from curative surgery in patients with early-stage HCC to palliative/hospice care in patients with metastatic HCC. In most tertiary care centers in the US, a multidisciplinary liver tumor board has become the standard of care and a key component of best practice protocol for patients with HCC.
\end{abstract}

Keywords: multidisciplinary team, MDT, hepatocellular carcinoma, HCC

\section{Introduction}

The importance of a multidisciplinary team in the management of cancer was highlighted in the National Health Services breast screening program. ${ }^{1,2}$ The goal of the multidisciplinary team was to improve the clinical outcomes for specific tumors starting with breast cancer, ${ }^{3}$ and more recently, hepatocellular carcinoma (HCC). ${ }^{4} \mathrm{HCC}$ is the third leading cause of cancer-related deaths worldwide. ${ }^{5,6}$ The rising incidence of HCC is likely due to the increasing prevalence of chronic hepatitis $\mathrm{C}$ virus (HCV) infection. ${ }^{5,6}$ No single treatment strategy can be applied to all patients with HCC, necessitating a multidisciplinary approach to tailor a management plan based on tumor burden, extent

Correspondence: Aijaz Ahmed Division of Gastroenterology and Hepatology, Stanford University School of Medicine, 750 Welch Road, Suite 210, Palo Alto, CA 94304, USA

$\mathrm{Tel}+|650498569|$

$\mathrm{Fax}+\mathrm{I} 6504985692$

Email aijazahmed@stanford.edu 
of metastasis, severity of hepatic decompensation, comorbid medical conditions, functional status, cancer-related symptoms, and patient preference..$^{7-10}$ The core members of multidisciplinary liver tumor board include liver transplant/ hepatobiliary surgeons, hepatologists/gastroenterologists, oncologists, radiologists, interventional radiologists, pathologists, and primary care physicians. ${ }^{8}$ Furthermore, additional consultative services can be obtained based on the individual needs of the patients. The aim of this review is to highlight the significance of a multidisciplinary liver tumor board to optimize the management of HCC.

\section{Prompt diagnosis of HCC}

The clinical features of HCC may be subtle and/or nonspecific, and a delay in the diagnosis of HCC may lead to poor outcomes. ${ }^{11}$ In patients with cirrhosis, symptoms associated with HCC may overlap with end-stage liver disease; therefore, the importance of HCC screening and surveillance protocols must be discussed with patients with cirrhosis. To optimize patient care and improve clinical outcomes, patients with $\mathrm{HCC}$ can be managed by a multidisciplinary team, comprising of specialists with different roles, expertise, and functions. ${ }^{8-10}$ In patients with cirrhosis, hepatic decompensation can manifest as ascites, spontaneous bacterial peritonitis, hepatorenal syndrome, bleeding from gastroesophageal varices, and hepatic encephalopathy. ${ }^{4}$ Patients who develop sudden-onset hepatic decompensation in the setting of cirrhosis should undergo an abdominal imaging study to rule out HCC. It is important to highlight that screening for HCC in patients with cirrhosis followed by surveillance every 6 months increases the likelihood of early-stage HCC diagnosis with potential for curative options. On the contrary, late diagnosis is associated with poor prognosis. A multidisciplinary liver tumor board provides a platform to promptly and optimally deliver any form of therapy following the diagnosis of $\mathrm{HCC}$.

\section{Staging criteria for $\mathrm{HCC}$}

The Barcelona Clinic Liver Cancer (BCLC) is a system ${ }^{7,11-13}$ that has been externally validated ${ }^{7,14}$ and is currently endorsed by the American Association for the Study of Liver Diseases, the European Society for Medical Oncology, and the European Association for the Study of the Liver. ${ }^{5,15,16}$ The stages within the BCLC system are linked to tumor burden, presence or absence of metastasis, extent of metastasis, hepatic functional reserve/severity of hepatic decompensation, physical/functional status, cancer-related symptoms, and patient preference. In addition, the BCLC system provides treatment guidance and predicts outcomes. ${ }^{5-7}$ Patients classified as having early-stage HCC (BCLC-A), defined as a single nodule or three nodules less than $3 \mathrm{~cm}$ in diameter, are eligible for potentially curative therapies such as hepatic resection or liver transplantation. Patients with intermediate-stage HCC (BCLC-B), those asymptomatic with multinodular tumors and without vascular invasion or extrahepatic spread, are eligible for locoregional therapy. Those with advanced-stage HCC (BCLC-C), who are symptomatic or have evidence of vascular invasion/extrahepatic spread, are eligible for sorafenib palliative therapy. Finally, patients with terminalstage HCC (BCLC-D) have either severe cancer-related symptoms or severe hepatic decompensation (Child-Pugh class $\mathrm{C}$ ) and may receive symptomatic palliative treatment only (Figure 1). ${ }^{4}$ Treatment options and management plan should be defined by a multidisciplinary liver tumor board (Figure 2) based on BCLC classification. Clinical trials and other experimental protocols available at the institution should be reviewed and offered to candidates who meet the inclusion criteria.

\section{Etiology of liver disease and pathogenesis of HCC}

Hepatitis B virus (HBV), HCV, alcoholic cirrhosis, aflatoxin b, nonalcoholic steatohepatitis, and hemochromatosis are some well-known causes of chronic liver disease. ${ }^{17}$ It is important to understand that a significant proportion of chronic liver disease can progress to cirrhosis (stage 4 hepatic fibrosis). Patients with advance stage 3 to 4 hepatic fibrosis are at risk for developing HCC. However, chronic $\mathrm{HBV}$ infection may result in $\mathrm{HCC}$ in the absence of cirrhosis. Therefore, it is important to start surveillance for HCC in patients with chronic $\mathrm{HBV}$ infection earlier on even in the absence of cirrhosis.

\section{Role of tumor biology of HCC}

The genetic and molecular heterogeneity of HCC complicates the understanding of this complex malignancy. Only a small proportion of HCC progresses rapidly without responding to bridging and/or downsizing treatments. Hence, the multidisciplinary liver tumor board should make an effort to identify cases of HCC that have the potential to grow rapidly and offer individualized therapy and evaluation to undergo treatment in the context of a clinical trial. Whole exome and genome sequencing has been implemented in order to determine the most common genetic mutations involved in HCC. Some previously reported gene mutations include TP53, CTNNB1, MLL4, MLL, ARIDIa/2, and JAK1. ${ }^{18-20}$ Mutations 


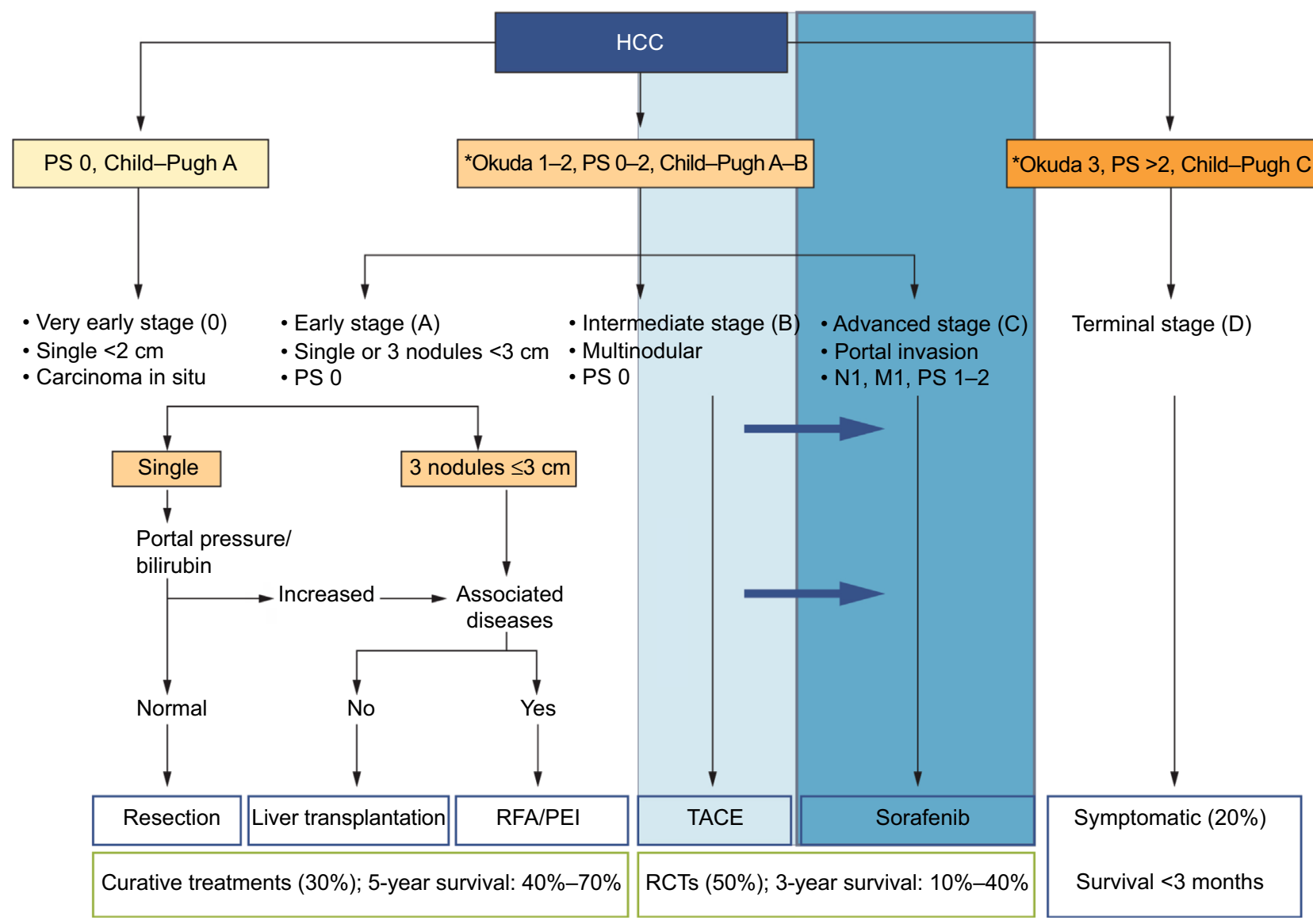

Figure I Barcelona Clinic Liver Cancer staging system.

Notes: Barcelona Clinic Liver Cancer stages: very early stage (0), early stage (A), intermediate stage (B), advanced stage (C), terminal stage (D). *Okuda: Okuda staging. NI and $\mathrm{MI}$ refer to TNM staging.

Abbreviations: HCC, hepatocellular carcinoma; PEl, percutaneous ethanol injection; PS, performance status; RCT, randomly controlled trial; RFA, radiofrequency ablation; TACE, transarterial chemoembolization.

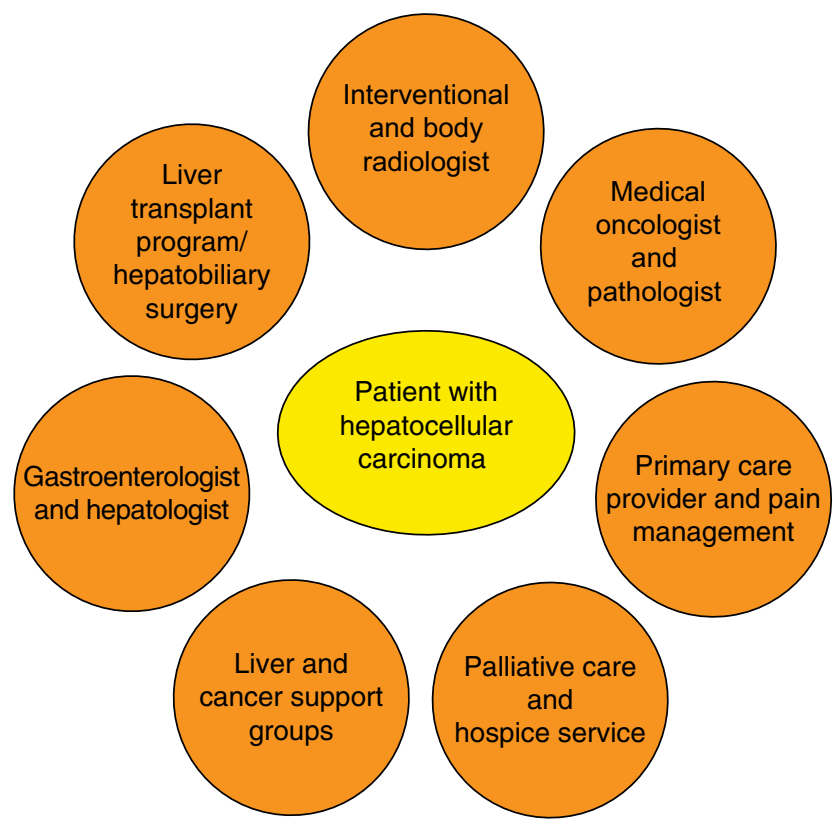

Figure 2 Multidisciplinary liver tumor board.

in p53 affect HCC recurrence, disease-free survival, and overall survival, which includes death from both HCC and the underlying liver disease. ${ }^{18}$ The prevalence of mutations in chromatin regulatory pathways further obscure the picture. Several studies have reported mutations in the switch/ sucrose nonfermenting-related ATP-dependent nucleosome remodelers: ARID1A and ARID2. ${ }^{19}$ It is important for the oncologist and pathologist to be aware of the research and clinical advances on a molecular level and if studies are available for enrollment.

\section{Improvement in treatment options for HCC}

Liver transplantation was a controversial treatment option for $\mathrm{HCC}$ in the early years until Mazzaferro et $\mathrm{al}^{21}$ described $75 \%$ 4-year survival in a cohort of patients with HCC limited to a single tumor lesion $\leq 5 \mathrm{~cm}$ or up to three tumor lesions, none greater than $3 \mathrm{~cm} .{ }^{22}$ This criteria, now known as the Milan criteria (Figure 3), has become the HCC listing criteria for liver transplantation. It has been shown that involvement of a multidisciplinary tumor board is associated with an improved 5 -year survival in colorecta ${ }^{23}$ and esophageal cancers ${ }^{24}$ and with an improved 2-year survival in head and neck cancers. ${ }^{25,26}$ Locoregional treatment modalities play a critical role in the 


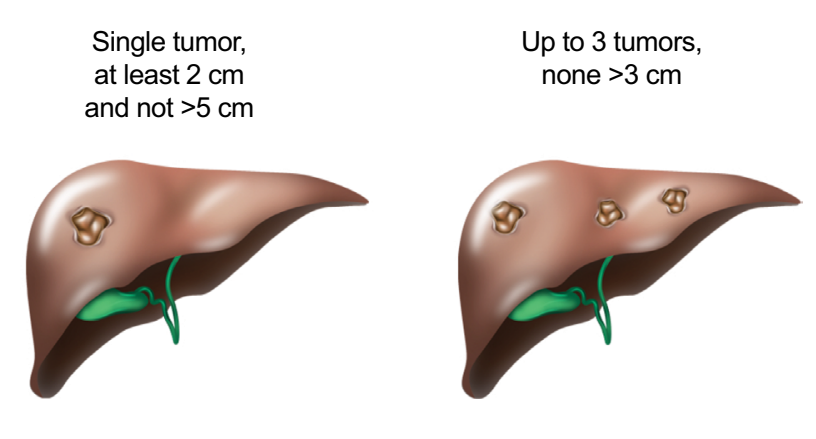

In the absence of

1) Macroscopic vascular invasion

2) Extrahepatic spread

- 5-year survival with transplantation: $~ 70 \%$

- 5-year recurrence rates: $<15 \%$

Figure 3 Milan criteria for selecting patients for liver transplants.

management of early or intermediate stage $\mathrm{HCC}$ via control of disease progression, tumor downstaging, and offering a bridge to liver transplantation. In advanced stages of the disease, the main aim is to control symptoms, prolong survival, and improve quality of life. Interventional radiology and surgery play a pivotal role in every stage of disease, with several options including direct ablation, portal vein embolization, transarterial embolization, transarterial chemoembolization, drug-eluting beads, and transarterial radioembolization. ${ }^{27}$ In each case, the rapid transfer of clinical knowledge among members of different specialties is essential to enhance the efficiency of treatment delivery and outcome. Long-term survival in patients with HCC diagnosed by screening improved significantly from 1998 to 2004 compared with the period from 1991 to 1997 due to the management of small HCC with liver transplantation and radiofrequency ablation. ${ }^{28}$

\section{Impact of a multidisciplinary approach}

The clinical decision making during the evaluation by the multidisciplinary liver tumor board relies heavily on the accuracy of diagnosis and staging of $\mathrm{HCC}$ before outlining a treatment plan. The diagnosis of HCC is established either by dynamic imaging involving radiologists using contrast enhancement and washout and/or by immunohistochemical methods employed by expert pathologists, especially for atypical nodules in both cirrhotic and noncirrhotic patients..$^{29,30}$ The 2010 American Association for the Study of Liver Diseases guidelines highlight the critical role of radiologists in the diagnosis of hepatic lesions that have a high pretest probability of HCC. ${ }^{10}$ The consensus statement on the histologic diagnosis of early-stage HCC from the
International Consensus Group for Hepatocellular Neoplasia defined the characteristics of low- and high-grade dysplastic nodules. ${ }^{31}$ A multidisciplinary approach ensures that HCC is accurately staged and that treatment recommendations are evidence-based, patient-centered, and reached by consensus. ${ }^{32}$ Specialists with different roles, expertise, and functions are required to manage $\mathrm{HCC}$ due to its complex genetic, pathological, etiological, and oncological features. ${ }^{8-10}$ The contribution of each team member varies and depends on the institution-specific expertise and the stage of $\mathrm{HCC}$ at diagnosis. ${ }^{33}$ The BCLC classification forms the backbone for clinical decision making and facilitates an optimal and individualized management plan for patients with HCC. . $^{34,35}$ Rapid transfer of clinical information and seamless communication is required between members of the multidisciplinary liver tumor board, ${ }^{8}$ along with frequent (preferably on a weekly basis) joint conferences to discuss management plans. ${ }^{36}$ Treatment strategies for HCC employed in the setting of a multidisciplinary liver tumor board have demonstrated several advantages, such as doubling of patients referred for treatment, patients presenting with early-stage HCC, curative treatment proportions increasing from $6 \%$ to $19 \%$, and palliative therapy increasing from $31 \%$ to $45 \% .{ }^{35}$ Cirrhosis is evaluated using the Child-Turcotte-Pugh score/classification $^{37}$ and the model for end-stage liver disease score. ${ }^{38}$ The complexity of these scoring and staging systems calls for interaction of various specialties at different stages of the disease. Gastroesophageal varices, ascites, spontaneous bacterial peritonitis, hepatorenal syndrome, and hepatic encephalopathy often coincide with treatment of HCC, both during curative therapy and palliative care. Early recognition and treatment of each of these concurrent complications are vital for improving outcomes. Published literature have also backed the idea that an integrated multidisciplinary approach can help optimize the management of HCC patients. ${ }^{5,10}$ Published data have supported the concept of a multidisciplinary approach in improving patient experience..$^{32}$

At our institution, we have had a multidisciplinary team for the management of $\mathrm{HCC}$ for the last 15 years, and the multidisciplinary approach to our patients with HCC is represented by Figure 2. A multidisciplinary liver tumor board optimizes the management of HCC patients by efficiently performing comprehensive consultations and developing their care plan. Our approach has been extremely convenient to patients and their families who at times travel from outreach clinics to our tertiary care center. In addition, the multidisciplinary approach helps physicians learn and review different protocols (experimental and standard of care) that are available. A multidisciplinary approach is the best practice 
and the standard of care at major tertiary care centers in most developed and developing countries.

\section{Conclusion}

In patients with $\mathrm{HCC}$, the rising incidence, genetic heterogeneity, multiple etiologies, and concurrent chronic liver diseases make diagnosis, staging, and selection of treatment options challenging. A multidisciplinary liver tumor board for HCC is beneficial in multiple ways. It can improve the management of patients through comprehensive and efficient consultations and through an offering of various options that are tailored on a case-by-case basis. In addition, a multidisciplinary approach offers physicians and other health care providers the opportunity to learn and review the variety of different protocols, experimental and/or approved, which are suitable for their patients. Furthermore, a multidisciplinary approach helps coordinate multiple clinic visits and scheduling for patients with advanced liver disease who are traveling long distances to their main tertiary care centers. In summary, a multidisciplinary approach for the management of HCC is the best practice and the standard of care at major tertiary care centers.

\section{Disclosure}

The authors report no conflicts of interest in this work.

\section{References}

1. Calman-Hine Report. A report by the expert advisory group on cancer to the chief medical officers of England and Wales. A Policy Framework for Commissioning Cancer Services - The Calman-Hine Report. London, UK: Department of Health; 1995.

2. Richards MA, Baum M, Dowsett M. Provision of breast services in the UK: the advantages of specialist breast units. London, UK: British Breast Group; 1994.

3. Cancer Guidance Sub-Group of the Clinical Outcomes Group. Improving outcomes in breast cancer. London, UK: Department of Health; 1996.

4. Barone C, Koeberle D, Metselaar H, et al. Multidisciplinary approach for HCC patients: hepatology for the oncologists. Ann Oncol. 2013;24(Suppl 2):15-23.

5. European Association for the Study of the Liver, European Organization for Research and Treatment of Cancer. EASL-EORTC Clinical Practice Guidelines: management of hepatocellular carcinoma. J Hepatol. 2012;56(4):908-943.

6. Cabibbo G, Rolle E, De Giorgio M, et al; HCC Working Group. Management of cirrhotic patients with hepatocellular carcinoma treated with sorafenib. Expert Rev Anticancer Ther. 2011;11(12):1807-1816.

7. Lencioni R, Chen XP, Dagher L, et al. Treatment of intermediate/ advanced hepatocellular carcinoma in the clinic: how can outcomes be improved? Oncologist. 2010;15(Suppl 4):42-52.

8. Gish RG, Lencioni R, Di Bisceglie AM, et al. Role of the multidisciplinary team in the diagnosis and treatment of hepatocellular carcinoma. Expert Rev Gastroenterol Hepatol. 2012;6(2):173-185.

9. Guy J, Kelley RK, Roberts J, et al. Multidisciplinary management of hepatocellular carcinoma. Clin Gastroenterol Hepatol. 2012;10(4):354-362.

10. Sherman M, Burak K, Maroun J, et al. Multidisciplinary Canadian consensus recommendations for the management and treatment of hepatocellular carcinoma. Curr Oncol. 2011;18(5):228-240.
11. Llovet JM, Brú C, Bruix J. Prognosis of hepatocellular carcinoma: the BCLC staging classification. Semin Liver Dis. 1999;19:329-338.

12. Llovet JM, Di Bisceglie AM, Bruix J, et al. Design and endpoints of clinical trials in hepatocellular carcinoma. JNatl Cancer Inst. 2008;100:698-711.

13. Forner A, Reig ME, de Lope CR, et al. Current strategy for staging and treatment: the BCLC update and future prospects. Semin Liver Dis. 2010;30:61-74.

14. Marrero JA, Fontana RJ, Barrat A, et al. Prognosis of hepatocellular carcinoma: comparison of 7 staging systems in an American cohort. Hepatology. 2005;41:707-716.

15. Bruix J, Sherman M; Practice Guidelines Committee, American Association for the Study of Liver Diseases. Management of hepatocellular carcinoma. Hepatology. 2005;42:1208-1236.

16. Bruix J, Sherman M, Llovet JM, et al. Clinical management of hepatocellular carcinoma. Conclusions of the Barcelona-2000 EASL conference. European Association for the Study of the Liver. J Hepatol. 2001;35:421-430.

17. Parikh P, Malhotra H, Jelic S, ESMO Guidelines Working Group. Hepatocellular carcinoma: ESMO clinical recommendations for diagnosis, treatment and follow-up. Ann Oncol. 2008;19(Suppl 2):27-28.

18. Cleary SP, Jeck WR, Zhao X, et al. Identification of driver genes in hepatocellular carcinoma by exome sequencing. Hepatology. 2013;58:1693-1702.

19. Fujimoto A, Totoki Y, Abe T, et al. Whole-genome sequencing of liver cancers identifies etiological influences on mutation patterns and recurrent mutations in chromatin regulators. Nat Genet. 2012;44:760-764.

20. Kan Z, Zheng H, Liu X, et al. Whole-genome sequencing identifies recurrent mutations in hepatocellular carcinoma. Genome Res. 2013;23:1422-1433.

21. Mazzaferro V, Regalia E, Doci R, et al. Liver transplantation for the treatment of small hepatocellular carcinomas in patients with cirrhosis. N Engl J Med. 1996;334:693-699.

22. Yao FY, Bass NM, Nikolai B, et al. Liver transplantation for hepatocellular carcinoma: analysis of survival according to the intention-to-treat principle and dropout from the waiting list. Liver Transpl. 2002;8:873-883.

23. Morris E, Haward RA, Gilthorpe MS, et al. The impact of the CalmanHine report on the processes and outcomes of care for Yorkshire's colorectal cancer patients. Br J Cancer. 2006;95:979-985.

24. Stephens MR, Lewis WG, Brewster AE, et al. Multidisciplinary team management is associated with improved outcomes after surgery for esophageal cancer. Dis Esophagus. 2006;19:164-171.

25. Birchall M, Bailey D, King P, South West Cancer Intelligence Service Head and Neck Tumour Panel. Effect of process standards on survival of patients with head and neck cancer in the south and west of England. Br J Cancer. 2004;91:1477-1481.

26. Rimola A, Garcia-Tsao G, Navasa M, et al. Diagnosis, treatment and prophylaxis of spontaneous bacterial peritonitis a consensus document. J Hepatol. 2000;32:142-153.

27. Gadahadh R, Valenti D, Aljiffry M, et al. Surgery and interventional radiology collaborate on combination therapy in hepatocellular carcinoma. US Gastroenterol Hepatol Rev. 2011;7:44-49.

28. Chan AC, Poon RT, Ng KK, et al. Changing paradigm in the management of hepatocellular carcinoma improves the survival benefit of early detection by screening. Ann Surg. 2008;247(4):666-673.

29. Trojan J, Zangos S, Schnitzbauer AA. Diagnostics and treatment of hepatocellular carcinoma in 2016: standards and developments. Visc Med. 2016;32(2):116-120.

30. Bruix J, Sherman M, American Association for the Study of Liver Diseases. Management of hepatocellular carcinoma: an update. Hepatology. 2011;53:1020-1022.

31. International Consensus Group for Hepatocellular Neoplasia. Pathologic diagnosis of early hepatocellular carcinoma: a report of the international consensus group for hepatocellular neoplasia. Hepatology. 2009;49:658-664.

32. Taylor C, Munro AJ, Glynne-Jones R, et al. Multidisciplinary team working in cancer: what is the evidence? Br Med J. 2010;340:c951.

33. Mazzaferro V, Majno P. Principles for the best multidisciplinary meetings. Lancet Oncol. 2011;12(4):323-325. 
34. Lange M, Abhari BA, Hinrichs TM, Fulda S, Liese J. Identification of a novel oxidative stress induced cell death by sorafenib and oleanolic acid in human hepatocellular carcinoma cells. Biochem Pharmacol. 2016;118:9-17.

35. Yan SY, Zhang Y, Sun C, et al. The clinical effect and relevant mechanism of combined sorafenib and radiofrequency ablation in the treatment of early small hepatocellular carcinoma. Oncol Lett. 2016;12(2):951-955.

36. Chang TT, Sawhney R, Monto A, et al. Implementation of a multidisciplinary treatment team for hepatocellular cancer at a Veterans Affairs Medical Center improves survival. HPB (Oxford). 2008;10(6):405-411.
37. Garcia-Tsao G, Lim JK; Members of Veterans Affairs Hepatitis C Resource Center Program. Management and treatment of patients with cirrhosis and portal hypertension: recommendations from the Department of Veterans Affairs Hepatitis C Resource Center Program and the National Hepatitis C Program. Am J Gastroenterol. 2009;104(7): 1802-1829.

38. MELD/PELD Calculator Documentation. United Network for Organ Sharing. Available from: www.unos.org/docs/MELD_PELD_Calculator_Documentation.pdf. Accessed September 5, 2016.
Journal of Multidisciplinary Healthcare

\section{Publish your work in this journal}

The Journal of Multidisciplinary Healthcare is an international, peerreviewed open-access journal that aims to represent and publish research in healthcare areas delivered by practitioners of different disciplines. This includes studies and reviews conducted by multidisciplinary teams as well as research which evaluates the results or conduct of such teams or health

\section{Dovepress}

care processes in general. The journal covers a very wide range of areas and welcomes submissions from practitioners at all levels, from all over the world. The manuscript management system is completely online and includes a very quick and fair peer-review system. Visit http://www.dovepress.com/ testimonials.php to read real quotes from published authors.

Submit your manuscript here: https://www.dovepress.com/journal-of-multidisciplinary-healthcare-journal 